\title{
Moving from reactive to proactive patient safety training in the future hospital: making learning a normal part of the working day
}

\author{
Authors: Sam Murray, Valerie Dimmock, Deblina Dasgupta, Haresh Mulchandani, Eleanor Wood \\ and David Watson
}

\section{Aims}

To develop regular simulation training on the acute care unit (ACU) for the multidisciplinary medical emergency team.

\section{Methods}

All simulations were performed using the iSimulate ALSi (iSimulate, Australia), which acted as the patient monitor, and a Resusci Anne mannequin (Laerdal Medical, Norway). The oncall medical registrar was invited to participate with a nurse and junior doctor. Participants were briefed beforehand. Training was explained to patients in nearby bed spaces. Eight scenarios were developed based around clinical incidents and mapped to relevant curricular objectives. Sessions were kept short, minimising the impact to the working day. Feedback using an anonymised paper-based questionnaire was collected from trainees and observing patients to better understand the public's perception of this training.

\section{Results}

Between November 2013 and July 2014, 24 weekly in situ simulation sessions were delivered in the ACU. On two occasions, there were no free beds or available staff to carry out the training. Sessions required one member of faculty to run the scenario and facilitate team debriefing. $63 \%$ of scenarios were led by the medical registrar. Nurses and foundation doctors were involved in all simulation sessions, totalling 78 multidisciplinary training experiences.

$100 \%$ of staff agreed that the training was relevant to their practice and beneficial to train within their usual working environment. $100 \%$ valued the opportunity to learn within their multidisciplinary teams. Fewer than $10 \%$ thought that the training had been disruptive to their work duties that day.

28 patients and visitors provided feedback. Over 50\% were already aware that healthcare professionals learnt using simulation. $100 \%$ were reassured knowing that the staff caring for them were undertaking this type of training.
In situ simulation training identified clinical governance issues, gaps in knowledge and unavailable medications and equipment. These included a lack of awareness of trust policies (eg a new major haemorrhage protocol), missing oxygen connectors and emergency drugs not available on the resuscitation trolley.

\section{Conclusions}

In situ simulation is a feasible method to deliver regular training in a busy work environment. Patients are supportive of this type of teaching. Running sessions regularly allows scenarios to be tailored to provide responses to clinical incidents. New guidelines/policies can be incorporated to keep staff updated so that important learning is disseminated. Delivering training within the workplace ensures that learning is transferable to the real world. It identifies latent errors in clinical environments, preventing potential harm before it occurs. This reflects a development in patient safety culture whereby training is not just reactive but proactive.

\section{Conflict of interest statement}

None. 